\section{Exploring the Utilization of Small Unmanned Aerial Vehicles (UAV) Known as Drones in Early Phase Disaster Response}

Dr. Jenifer Luman MD, MPH, FACEP ${ }^{1}, M r$. Benjamin Luman $P A-C^{1}$

1. Luman Medical, Encinitas, United States

2. University of California, Irvine, United States

Introduction: Disaster Medical Response is a challenging field where opportunities for advancement are welcomed. Small Unmanned Aerial Vehicle (sUAV) technology (i.e., drones) has made enormous strides in the past few years and is poised for utilization in the early disaster response phase.

Aim: To discuss current uses of UAVs, proposed utilization and logistical details, technological advancements, current deficits, and training.

Methods: Our Foundation, Luman Medical, is working in the field of UAV integration for small to large scale disaster response. The concept is to equip first responders with small, relatively inexpensive, programmable drones that come equipped with hardware and software that are easy to use for inexperienced as well as skilled sUAV pilots.

Discussion: These UAVs could increase ease and speed of deployment for early assessments of disaster area mapping, thermal imaging, ingress and egress routes, the discovery of survivors, communications, and delivery of supplies. Drone technology offers a new and growing type of tool in the disaster response arena. It is our hope to explore an integration that is easy, safe, and affordable to augment and enhance existing disaster response planning.

Prehosp Disaster Med 2019;34(Suppl. 1):s132 doi:10.1017/S1049023X19002875

First Aid Management of Hypothermia and Cold Injuries Dr. Rowena Christiansen ${ }^{1,2,3,4}$

1. The University of Melbourne, Parkville, Australia

2. Ormond College, The University of Melbourne, Parkville, Australia

3. The Australian Resuscitation Council, Melbourne, Australia

4. International Commission for Mountain Emergency Medicine (ICAR MEDCOM), Zurich, Switzerland

Introduction: Best practice first aid management of accidental hypothermia and cold injuries in the prehospital setting is relevant for disaster management in cold environments as well as for wilderness and expedition medicine, and space medicine. In the Australasian context, guidance is currently taken from the Australian Resuscitation Council (“ARC”) Guidelines dealing with these issues.

Aim: To review and update the recommendations of the ARC Guideline 9.3.3 Hypothermia: First-Aid Management (February 2009) and ARC Guideline 9.3.6 Cold Injury (March 2000).

Method: The review is being undertaken through a combination of a focused literature review and expert opinion. Through the author's membership of the International Commission for Alpine Rescue ("ICAR") Alpine Emergency Medicine Commission, two northern-hemisphere experts on hypothermia have reviewed the guidelines and provided commentary and recommendations.

Results: Much of the literature around accidental hypothermia and cold injuries (including frostbite, frostnip, and chilblains), relies on expert opinion and case studies. There are relatively few randomized controlled trials, and these are often confined to the laboratory setting. As a result, there is a heavier reliance on expert opinion than in any other areas of medicine.

Discussion: This presentation will summarize the current best practice recommendations for the first aid management of accidental hypothermia and cold injuries through combining the existing ARC Guidelines with key advances identified through the literature review, and the key management recommendations stemming from expert opinion. This will provide attendees with a cohesive set of clinical practice recommendations which can be used in the field.

Prehosp Disaster Med 2019;34(Suppl. 1):s132

doi:10.1017/S1049023X19002887

\section{Focused Ultrasound: Applications and Implications for} Education

Mr. Cameron Moore ${ }^{1,2}$, Professor Pamela Rowntree ${ }^{1,2}$

1. Queensland University of Technology, Brisbane, Australia

2. QUT Institute of Health and Biomedical Innovation, Brisbane, Australia

Introduction: Focused or point of care ultrasound applications have been integrated into prehospital assessments, triage capacities, military applications, trauma, and emergency health care settings, and medical school curriculums. Often, the inclusion of focused ultrasound is to answer specific clinical questions. However, the value is ultimately determined by the experience, skills, and training of the operator performing and interpreting the examination. Ultrasound was reserved for traditional imaging providers as little as two decades ago. However, as the application of ultrasound expands within clinical medicine, there is an increasing necessity for associated education and training.

Aim: To highlight the applications and uses of focused ultrasound in the current diverse health care landscape while identifying the associated educational considerations, including the undergraduate tertiary education sector.

Methods: A search of peer-reviewed published literature was undertaken to determine the range of current usage of ultrasound imaging across professions, and to identify the education and training available.

Results: The results discussed within this presentation will highlight identified trends, ultrasound applications, educational considerations, and potential future practices based on the content of the literature explored.

Discussion: Technology is rapidly advancing in the field of medical ultrasound with handheld ultrasound scanners now smaller, less expensive, and more accessible than ever before. Paralleled with these advances and the more generous use of ultrasound come the expectation and pressures of competent skill diversity among healthcare staff and specialists. Significantly, sonography is still considered by many as the most technically demanding and operator dependent medical 